\title{
Research Paper: The Role of Psychiatric Symptoms, Social Support and Meaning in Life in Predicting Internet Addiction Among University Students: A Causal Model
}

\author{
Mohammad Ghaderi Rammazi ${ }^{1}$, Ghasem Askarizadeh², Gholamreza Ahmadi ${ }^{* *}$, Kouros Divsalar $^{3}$ \\ 1. Department of Psychology, School of Education and Psychology, Isfahan University, Isfahan, Iran. \\ 2. Department of Psychology, School of Literature and Humanities, Shahid Bahonar University, Kerman, Iran. \\ 3. Neuroscience Research Center, Institute of Neuropharmacology, Kerman University of Medical Sciences, Kerman, Iran.
}

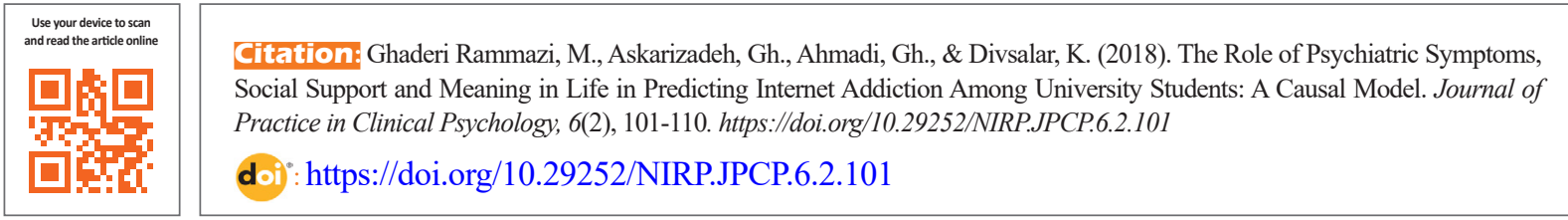

Article info:

Received: 14 Jun. 2017

Accepted: 29 Sep. 2017
Keywords:

Addiction, Internet, Model, Social support, Symptoms

\section{ABSTRACT}

Objective: The aim of this study was to investigate the direct and indirect roles of psychiatric symptoms, social support, and meaning in life in predicting internet addiction among university students.

Methods: The study was performed in a cross-sectional design by employing the path analysis to explore the model fitting. All the students at Kerman University of Medical Sciences constituted the research population. A total of 159 students were selected, by random cluster sampling method, as sample members. The questionnaires were utilized for data collection.

Results: The results revealed that more than half of the participants were afflicted with the Internet addiction or were on the verge of addiction. Correlation coefficients results indicated a positive correlation between all the subscales of psychiatric symptoms and students' Internet addiction and also a negative correlation between all the subscales of social support and Internet addiction. Moreover, a negative correlation was found between meaning in life (PML) and internet addiction. Among all the endogenous variables, depression accounted for most of the variances in Internet addiction. Social support and PML also accounted for a considerable part of the variances in Internet addiction, either directly or indirectly. In addition, the goodness of fit indices was indicative of an acceptable fitness ( $\mathrm{CFI}=0.96, \mathrm{NFI}=0.94, \mathrm{IFI}=0.92$, RMSEA $=0.17$ ).

Conclusion: Considering the fit indices, path coefficients, and the significance level, it can be maintained that the presented model for Internet addiction has an acceptable goodness of fit and that it explains $75 \%$ of the variance in participants' Internet addiction.

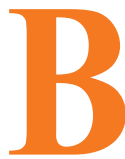

\section{Introduction}

ased on the latest report of The Global

Internet Society, the population of Inter- net users has tripled over the current decade (Brown, 2017). This outstanding spurt of the Internet use and its penetration into human lives has led to more reliance on the Internet, and the risk of becoming addicted to

\section{* Corresponding Author:}

Gholamreza Ahmadi, MSc.

Address: Neuroscience Research Center, Institute of Neuropharmacology, Kerman University of Medical Sciences, Kerman, Iran.

Tel: +98 (34) 32264180

E-mail: gholamrezaahmadi10@yahoo.com 
it has increased more than ever before (Pontes, Szabo, \& Griffiths, 2015). As per the existing definitions, the term "Internet addiction" refers to any "online-related, compulsive behavior interfering with normal life, and being a cause of severe stress for family, friends, beloved, and one's work environment" (Lee, Shin, Cho, \& Shin, 2014; Xu et al., 2012). Therefore, nowadays, Internet addiction has turned into one of the most preferred issues among health authorities (Lee et al., 2014; $\mathrm{Xu}$ et al., 2012). Based on previous research, the prevalence rate of Internet addiction has been assessed at 9.8 to $15.2 \%$ in the United States, 1.5 to $19.4 \%$ in Greece, $15.3 \%$ in Taiwan, 2.6 to $14.9 \%$ in South Korea (Lee et al., 2014) and 26.8\% in Iran (Moiedfar, Habib Poor, \& Ganji, 2005). Various studies have shown that there is a relationship between Internet addiction and major psychiatric symptoms like depression, anxiety, OCD, drug abuse, ADHD, sleeping disorders, smoking, drinking and risky sexual relations (Alavi, Maracy, Jannatifard, Eslami, \& Haghighi, 2010; Ghanbari, Amani, Namdari Pezhman, Bidi, Kareshki, 2012; Jafari \& Fatehizadeh, 2012; Khoshakhlagh \& Faramarzi, 2012; Ko, Yen, Yen, Chen, \& Chen, 2012; Koukia, Mangoulia, \& Alexiou, 2014; Mazhari, 2012; Pirzadeh, 2012; Sung, Lee, Noh, Park, \& Ahn, 2013).

Social support has a valuable share in relieving the mental health problems and maintaining the psychological well-being (Mincu \& Taşcu, 2015). It has been found that disrupting one's real social relationships can reinforce a person's tendency toward using cyberspace of Internet to gain the required social support (Esen \& Gündoğdu, 2010). The results of different studies have revealed that students with Internet addiction have problems in achieving social support and most of the times they get lower scores in social support tests (Onsroodi, Hoseinian, Salehi, \& Moemeni, 2014; Tang, Chen, Yang, Chung, \& Lee, 2016; Wu, 2004).

Meaning in life, which is defined as "being purposeful, organized and finding the sense of worth in one's life" (Abeyta, Routledge, Juhl, \& Robinson, 2015; Steger, Fitch-Martin, Donnelly, \& Rickard, 2014) interferes with the most of the variables connected to a human's mental health in the difficult situations. Regarding the fact that some types of human's mental problems arise out of a person's inability to find meaning in his/her life (Kleftaras \& Psarra, 2012), it can be speculated that lack of meaning in life expedites people's penchant for entering into the cyber world and consequently aids Internet addiction. Unfortunately, studies show that despite the importance of some psychological variables such as being under the influence of psychiatric symptoms as a harmful factor or receiving needful social support or even having a meaning in life that helps people to motivate themselves in life, overwhelming situations and vicissitudes which can be termed as a protective factor, have been neglected in the most of the studies.

Thus, considering the provided discussion and the fact that the direct and indirect relationships between variables related to Internet addiction have not been investigated (especially in a causal model), the present study was aimed to examine and explore the direct and indirect relationship between the psychiatric symptoms, social support, meaning in life and Internet addiction as well as in predicting the internet addiction among university students. In addition, it required an answer to the question: Does the presented model have an acceptable goodness of fit for predicting students' Internet addiction?

\section{Methods}

The present study was descriptive in nature and was performed in a cross-sectional design using path analysis. As fifteen subscales existed in the current study (nine for psychiatric symptoms, four for social support, and two for meaning in life), and as a sample size of 10 members per each subscale was recommended in the path analysis studies (Hooman, 2012), a sample size of 150 subjects was the requisite for performing this study. The research population consisted of all male and female students of Kerman University of Medical Sciences (Southeastern Iran; for academic year 2014-15). Considering a probable sample drop out, initially, 170 subjects were selected as sample members and a Multicluster sampling method was followed. Therefore, out of the nine medical-sciences faculties in Kerman city, five faculties were randomly selected. Subsequently, one field of study from each faculty and one classroom from each field were randomly selected as the sampling unit.

Having made the required arrangements with university authorities, the authors referred to the students' classrooms and distributed the questionnaires among the subjects, asking them to fill out the questionnaires. Participation in the study was completely arbitrary, and an informed consent was obtained from all the subjects. Spending at least one semester of studying at college and answering the questions honestly was the inclusion criteria. After collecting the questionnaires, 159 questionnaires were qualified, and 11 questionnaires were removed due to non completion. The qualified questionnaires were analyzed through AMOS and SPSS software (v. 22), using a path analysis technique, to achieve the main aim of the study and to assess the model fitting. The following instruments 
were used for data collection: Young's Internet Addiction Test (IAT), The Symptoms Checklist Questionnaire (SCL-90-R), Social Support Questionnaire (SSQ), and Meaning in the Life Questionnaire (MLQ).

In Young's Internet Addiction Test (IAT), developed by Young (1988; cited by Alavi et al., 2010), the participants were expected to answer each of the 20 items on a five-point Likert scale. The test scores range from 0 to 100 , where higher scores represent an individual's greater dependence on the Internet. In an earlier study, Young and his colleges reported the internal reliability of the questionnaire as 0.92 and maintained that the questionnaire enjoyed a good face validity (Alavi et al., 2010). In Iran, the Cranach's coefficient alpha of the questionnaire was assessed at 0.71 for reliability. The discriminant and concurrent validities of the questionnaire were assessed at 0.62 and 0.50 , respectively (Masoudnia, 2012). In this study, using Cronbach's alpha coefficient, the internal consistency was assessed at 0.78 as the total score of Young's Internet Addiction.

The Symptoms Checklist Questionnaire (SCL-90-R) is a self-reporting instrument, which is used for assessing the individual psychiatric symptoms for clinical or scholarly purposes. It has been developed by Deragotis, Lipman, and Covi, 1973, initially (Sharifi, Shokr Kon, Ahadi, \& Mazaheri, 2009). The 90 items of the questionnaire are used to assess the nine dimensions of psychiatric symptoms. Each item is scored on a 5-point Likert scale ranging from 0 to 4 (Derogatis \& Melisaratos, 1983). Sharifi, et al. (2009) investigated the reliability of the instrument by using the split-half method and reported the coefficients between 0.76 to 0.89 for the sub-scales and 0.76 for the total score. To assess the validity of the instrument, the researchers correlated it with General Health Questionnaire (GHQ) and reported the coefficient correlation at 0.49 (Sharifi et al., 2009). The internal consistency (assessed via Cornbrash's alpha coefficient) was equal to $0.79,0.78,0.91,0.89,0.75,0.79,0.76,0.76$, and 0.78 , respectively for: somatization, obsessive- compulsive, interpersonal sensitivity, depression, anxiety, hostility, phobia, paranoid thinking and psychoticism. The total score of the questionnaire was obtained as 0.78 .

Social Support Questionnaire (SSQ) is a self-reporting questionnaire that, initially, was constructed by Wax, et al. (1986), (cited by Asgari \& Sharaf Al-din, 2008). From the 23 items in the questionnaire, 8 items measure family social support, 7 items deal with social support from friends, and the remaining 8 items measure the social support from others (Asgari \& Sharaf
Al-din, 2008). For the sake of determining validity, the total score for the questionnaire was correlated with that of the social anxiety questionnaire, so the correlation was reported as -0.17 , indicating the good validity of the instrument. Moreover, the reliability coefficient of the instrument was calculated at 0.72 , through Cranach's coefficient alpha, and at 0.70 , through test-retest methods (Mashak, 2006). Based on the calculations of this study, the internal consistency for family, friends, others, and total social support was equal to $0.77,0.78$, 0.79 and 0.78 , respectively.

Meaning in the Life Questionnaire (MLQ) was constructed by Steger, Frazier, Oishi, \& Kaler (2006). It contains 10 items on a seven-point Likert scale (Steger, et al., 2006) and measures two sub-scales of the Presence of Meaning (PML) and Searching of Meaning (SML). The internal consistency coefficients for the subscales have been calculated at 0.81 and 0.84 for PML and SML respectively. High construct and convergent validities also have been reported for this questionnaire through studies conducted in Iran (Mesrabadi, Ostovar, \& Jafarian, 2013). In the present study, using Cornbrash's alpha coefficient, the internal consistency was assessed at 0.86 and 0.84 for PML and SML, respectively.

\section{Results}

One hundred fifty-nine students consisting of 92 males $(58 \%)$ and 67 females (42\%), with ages ranging from 18 to 34 and mean ages of 22.6, were investigated in the current study. These values and the other participants' demographic data are provided in Table 1. As Table 1 shows, $25.12 \%$ of the students were studying Public Health, 28.93\% were studying Nursing and Midwifery, 20.75\% were studying Management and Information Science, $17.61 \%$ were studying Medicine, and 7.54\% were studying Dentistry. $70.44 \%$ of the students were dwelling in dormitories, and the others were living elsewhere. Table 1 also shows that the participants' mean and standard deviations for Internet addiction scores were 57.24 and 24.93 respectively. The Internet addiction scores of $45.9 \%$ of the students were normal (having scores below 50), $23.6 \%$ were at risk of addiction (having scores between 50 to 79 ), and $30.6 \%$ were severe Internet addicts (having scores between 80 and 100). The cut-off points have suggested by previous studies (e.g. Alavi et al., 2010). It means that more than half of the students were either at the risk of addiction or were severe Internet addicts.

Based on the study aims the direct relationships of psychiatric symptoms, social support and meaning in life with the students' Internet addiction scores had been 
Table 1. Participants' demographic data $(n=159)$

\begin{tabular}{|c|c|c|c|}
\hline & Characteristic & Mean (SD) & $\%$ \\
\hline \multirow{3}{*}{ Gender } & Male & & 58 \\
\hline & & & 2 \\
\hline & teritare & & \\
\hline \multirow{5}{*}{ Age } & $17-22$ & & 40.27 \\
\hline & $23-28$ & & 48.42 \\
\hline & & $22.6(7.9)$ & \\
\hline & $29-34$ & & 6.91 \\
\hline & $33-38$ & & 4.4 \\
\hline \multirow{5}{*}{ Field } & Public health & & 25.12 \\
\hline & Nursing and midwifery & & 28.93 \\
\hline & Management and information science & & 20.75 \\
\hline & Medicine & & 17.61 \\
\hline & Dentistry & & 7.54 \\
\hline \multirow{3}{*}{ Dwelling status } & Dormitory & & 70.44 \\
\hline & & & \\
\hline & Non-dormitory & & 29.56 \\
\hline \multirow{3}{*}{ Internet addiction status } & Normal user & & 45.9 \\
\hline & In the verge of addiction & $57.24(24.93)$ & 23.6 \\
\hline & Internet addicted & & 30.6 \\
\hline
\end{tabular}

calculated. Firstly it was found that, as shown in Table 2 , there was a positive correlation between all subscales of psychiatric symptoms and students' Internet addiction score $(\mathrm{P}<0.001)$. For example, somatization $(\mathrm{r}=0.653)$, obsessive-compulsive $(\mathrm{r}=0.565)$, interpersonal sensitivity $(\mathrm{r}=0.644)$, depression $(\mathrm{r}=0.638)$, anxiety $(\mathrm{r}=0.620)$, hostility $(\mathrm{r}=0.656)$, phobia $(\mathrm{r}=0.383)$, paranoid thinking $(\mathrm{r}=0.427)$, and psychoticism $(\mathrm{r}=0.509)$. Moreover, there was a negative correlation between social support and its sub-scales and students' Internet addiction score $(\mathrm{P}<0.001)$. For example, family social support $(\mathrm{r}=0.722)$, friends social support $(\mathrm{r}=-0.798)$, others social support $(\mathrm{r}=-0.691)$, and total social support $(\mathrm{r}=-0.345)$. In addition, while there was a negative correlation between PML and students' Internet addiction $(r=-0.320, P<0.001)$, the correlation between SML and students' Internet addiction was not significant $(\mathrm{r}=-0.63, \mathrm{P}<0.056)$. Secondly, to devise a causal model for students' Internet addiction and to take the direct and indirect relationships between endogenous and exogenous variables into account, path analysis method was applied. Prior to that, the assumptions of parametric tests were met. The Boxplot showed that there were some outliers in the anxiety, phobia and psychoticism subscales, so these scattered data were deleted. The outputs of Shapiro-wilk and Levene tests, which were conducted to examine the Normality of distribution and the Homogeneity of variances in Endogenous and exogenous variables, confirmed with the Normality of distribution and the Homogeneity of variances in the studied variables $(\mathrm{P}>0.05)$. After meeting the required assumptions of the analysis, the assumed model was evaluated using the Comparative Fit Index (CFI), Normed Fit Index (NFI) and Root Mean Square Error Approximation (RMSEA).

The Table 3 shows that $\mathrm{X}^{2}$ is significant $(\mathrm{P}<0.001)$, however, because of its high sensitivity to the sample size, the other indices with more attributability ought to be considered (Meyers, Gamst, \& Guarino, 2006). These indices were indicative of the suitable goodness of fit of the assumed model with the observed data $(\mathrm{NFI}=0.94$; $\mathrm{CFI}=0.96$ ). While the RMSEA was indicative of poor fitness of the Model, the considerable value of other indices confirmed the acceptable goodness of fit for the 
Table 2. Correlation coefficients among psychiatric symptoms, social support, meaning in life and the students' internet addiction

\begin{tabular}{|c|c|c|c|c|c|}
\hline & Scales & Correlation Coefficient & $\mathbf{P}$ & Mean & SD \\
\hline \multirow{9}{*}{ Psychiatric symptoms } & Somatization & 0.653 & $<0.001$ & 11.29 & 5.94 \\
\hline & Obsessive- compulsive & 0.565 & $<0.001$ & 9.80 & 4.81 \\
\hline & Interpersonal sensitivity & 0.644 & $<0.001$ & 8.41 & 4.39 \\
\hline & Depression & 0.638 & $<0.001$ & 12.95 & 6.32 \\
\hline & Anxiety & 0.620 & $<0.001$ & 9.75 & 5.08 \\
\hline & Hostility & 0.656 & $<0.001$ & 5.68 & 3.17 \\
\hline & Phobia & 0.383 & $<0.001$ & 2.57 & 2.11 \\
\hline & Paranoid thinking & 0.427 & $<0.001$ & 2.06 & 1.78 \\
\hline & Psychoticism & 0.509 & $<0.001$ & 3.52 & 7.79 \\
\hline \multirow{4}{*}{ Social support } & Family social support & -0.722 & $<0.001$ & 4.06 & 1.65 \\
\hline & Friends social support & -0.798 & $<0.001$ & 3.34 & 2.11 \\
\hline & Others social support & -0.691 & $<0.001$ & 3.75 & 1.81 \\
\hline & Total social support & -0.845 & $<0.001$ & 11.15 & 4.88 \\
\hline \multirow{2}{*}{ Meaning in life } & Presence of Meaning of Life (PML) & -0.320 & $<0.001$ & 19.78 & 7.43 \\
\hline & Search for Meaning of Life (SML) & -0.63 & $<0.056$ & 10.15 & 4.82 \\
\hline
\end{tabular}

Dependent variable: Internet addiction

obtained model. All path coefficients were significant statistically $(\mathrm{P}<0.001)$ or practically.

As shown in Figure 1, among the endogenous variables, depression explained most variance in Internet addiction $(\beta=0.69)$. Although the lowest path coefficients belonged to direct relationships between PML and depression $(\beta=-0.21)$ and anxiety $(\beta=-0.18)$ variables, PML significantly affected Internet addiction through these variables. Social support also explained a considerable part of Internet addiction variance either directly $(\beta=0.50)$ or indirectly (by imposing the moderating effects on anxiety and depression). Hostility also explained a great deal of social support variance directly $(\beta=-0.87)$ or indirectly (performing its mediating role for interpersonal sensitivity, obsessive-compulsive and somatization). These mediating variables were themselves explained by anxiety $(\beta=0.62)$. Moreover, more than half of the depression variance was explained by anxiety $(\beta=0.53)$. In addition to what was mentioned above, evaluating the Squared Multiple Correlations showed that the model variables had been accounted for by $75 \%$ of the explained variance in students' Internet addiction. Based on these coefficients, $55 \%$ of depression variance was explained by PML, social support, and anxiety and $40 \%$ of anxiety variance was explained by social support and PML. Therefore, based on the indices of fitness and significance levels of the paths it can be claimed that the obtained model has an adequate fitting for the data.

\section{Discussion}

The present study was conducted with the main aim of investigating the direct and indirect roles of psychiatric

Table 3. The indices of fitness of the model

\begin{tabular}{|c|c|c|c|c|c|c|}
\hline$x$ & df & $\mathbf{P}$ & CFI & NFI & IFI & RMSEA \\
\hline 199049 & 35 & 0.000 & 0.96 & 0.94 & 0.92 & 0.17 \\
\hline
\end{tabular}




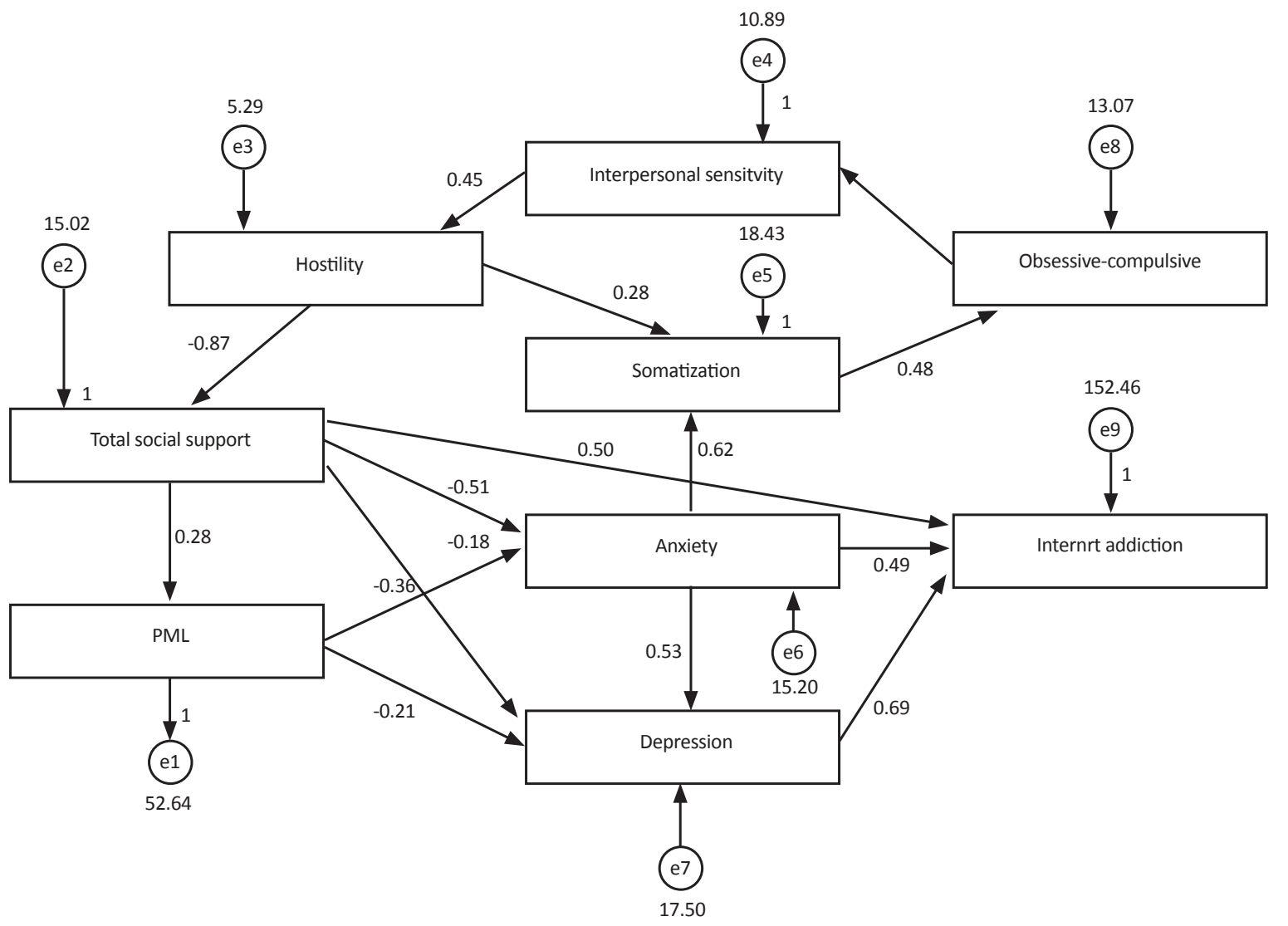

Figure 1. Obtained model along with path coefficients

symptoms, social support, and meaning in life in predicting Internet addiction among university students and also devising a causal model for students' Internet addiction. The results initially revealed the high prevalence of Internet addiction among students, as some other studies did (Alavi et al., 2010; Khoshakhlagh \& Faramarzi, 2012; Koukia et al., 2014; Lee et al., 2014; Moiedfar et al., 2005). Therefore, as noticed by other researchers (Pirzadeh, 2012), we believe that raising students' awareness on the destructive effects of the improper use of Internet is of paramount importance. Based on the study aims, the direct correlations between the variables were taken into account, and the results revealed that there was a positive correlation between all psychiatric symptoms and Internet addiction. This finding is in line with most previous studies (Alavi et al., 2010; Jafari \& Fatehizadeh, 2012; Khoshakhlagh \& Faramarzi, 2012; Ko et al., 2012; Koukia et al., 2014; Lee et al., 2014; Mazhari, 2012; Pirzadeh, 2012; Sung et al., 2013). However, despite the fact that most of the studies attest to the relationship between psychiatric symptoms and Internet addiction, the underlying causes still remain unknown. In other words, Internet addiction can be the cause or the consequence of a psychiatric symptom (Jafari \& Fatehizadeh, 2012) but regarding the clear relationship be- tween Internet addiction and the psychiatric symptoms it appears that there is (are) mechanism(s) that justify this reciprocal relationship (Ko et al., 2012). Thus, we need more studies to also cast light on the reciprocal relationships between Internet addiction and psychiatric symptoms (Koukia et al., 2014).

Based on the direct relationships, there was a negative correlation between social support and its sub-scales and students' Internet addiction. Moreover, social support explained a considerable part of Internet addiction variance, either directly or indirectly (imposing the moderating effects on PML, anxiety, and depression) and these findings are also in agreement with some previous studies (Onsroodi et al., 2014; Wu, 2004). To explain how failure to receive the required social support relates to Internet addiction, it can be maintained that social support is provided through consistency between one's social demands from others and their personal perception of the realized wants (Mincu \& Taşcu, 2015). Hence, when a person fails to receive social support in the real world, they tend to attract that social support from the cyber world (Mustafa, Short, \& Fan, 2015). On the other hand, the prevalence rate of Internet addiction is lesser in intimate and more supportive families because of the 
member's propensity for participating in enjoyable hobbies (such as talking to each other, eating, dining out, shopping, etc.). In such situations, children take less delight in using the Internet and receiving the social support from a cyber-world (Onsroodi et al., 2014).

Further, based on the direct correlation coefficients, there was a negative relationship between PML and students' Internet addiction. Although, at the moment, no study has been found on the relationship between meaning in life (including PML or SML) and Internet addiction, other studies indicate that there is a negative relationship between meaning in life and the wide range of psychiatric symptoms such as depression (Kleftaras \& Psarra, 2012; Owens, Steger, Whitesell, \& Herrera, 2009; Schulenberg, Strack, \& Buchanan, 2011; Woo \& Brown, 2013), anxiety (Schulenberg et al., 2011), PTSD (Owens et al., 2009; Schulenberg et al., 2011; Woo \& Brown, 2013) and feeling guilty (Owens et al., 2009). It means that the presence of meaningful goals in the life can help the students to beat their psychiatric problems and get rid of cyberspace affinities as well as troubles.

Besides the direct relationships, the obtained model also revealed some interesting relationships between the variables. In one of these relationships, the model illustrated that among psychiatric symptoms, depression, which can be termed as the best predictor of Internet addiction, is under the influence of social support and also PML. Obviously, as social support decreases, the feeling of loneliness increases. Loneliness, as a restricted mental status, causes the poorer perception of social support in quantity or quality and, in turn, creates a propitious condition for experiencing helplessness, spiritual emptiness. Eventually it pushes the person into other disorders, especially depression (He, Zhou, Li, Cao, \& Guan, 2014). The proposed model also sheds light on the fact that the relationship between PML and students' Internet addiction is influenced by the mediating role of depression and anxiety. It can be asserted that anxiety is not anything more than a pervasive and chronic fear of ambiguous stimulants (Brassai, Piko, \& Steger, 2010). Therefore, those people who do not have a clear meaning in their lives experience more degrees of ambiguous situations in their lives. Additionally, the role of depression as a moderating variable between PML and Internet addiction is essential because $d$ depression, as a sign of existential emptiness, makes people construct a meaning in their lives to overcome the feeling of emptiness (Frank1, 1977). Therefore, a depressed person inclines to the distinct domains in order to create a meaning for his/ her life, and the Internet cyber world, probably, is the most available and the easiest way to reach that target.
Therefore, considering the valuable findings of the present study and based on what has been discussed in the current section, it can be concluded that the model of the study has satisfactorily predictive power and can be used to predict Internet addiction among the students. It has also revealed some direct and indirect relationships among the variables. Thus, all experts are recommended to use the model in either their therapeutic or preventive actions.

However, aside from the mentioned benefits, the current study encountered some critical limitations, especially the small size of the sample and limiting the scope of research to the Kerman University of Medical Sciences, which led to some restrictions in the generalizability of the results to other populations. So, in future researches, it is recommended that larger samples be studied and regarding the importance of variables such as social support and meaning in life, practical actions for treatment of Internet addiction among the students be taken into consideration.

\section{Acknowledgements}

Participation of students was voluntary, which meant that they could withdraw from the study at any time. The authors wish to appreciate all students and other individuals, who kindly contributed to the completion of this research. This study was funded by Neuroscience Research Center, Institute of Neuropharmacology, Kerman University of Medical Sciences, Kerman, Iran. All authors equally contributed to the writing and revision of this manuscript.

\section{Conflict of Interest}

The authors declared no conflicts of interest.

\section{References}

Abeyta, A. A., Routledge, C., Juhl, J., \& Robinson, M. D. (2015) Finding meaning through emotional understanding: Emotional clarity predicts meaning in life and adjustment to existential threat. Motivation and Emotion, 39(6), 973-83. doi: 10.1007/s11031-015-9500-3

Alavi, S. S., Maracy, M. R., Jannatifard, F., Eslami, M., \& Haghighi, M. (2010). A survey of relationship between psychiatric symptoms and internet addiction in students of Isfahan Universities. Scientific Journal of Hamadan University of Medical Sciences, 17(2), 57-65.

Asgari, P., \& Sharaf Al-din, H. (2008). [Relationship between social anxiety, hope and social support with subjective well- 
being in postgraduate students of Khouzestan Science and Research Branchs (Persian)]. New Findings in Psychology, 3(9), 25-36.

Brassai, L., Piko, B. F., \& Steger, M. F. (2010). Meaning in life: Is it a protective factor for adolescents' psychological health. International Journal of Behavioral Medicine, 18(1), 44-51. doi: 10.1007/s12529-010-9089-6

Brown, K. (2017). Global internet report 2016. Washington D.C.: Internet Society.

Derogatis, L. R., \& Melisaratos, N. (1983). The brief symptom inventory: An introductory report. Psychological Medicine, 13(3), 595. doi: $10.1017 / \mathrm{s} 0033291700048017$

Esen, B. K., \& Gündoğdu, M. (2010). The relationship between internet addiction, peer pressure and perceived social support among adolescents. International Journal of Educational Researchers, 1(2), 29-36.

Frankl, V. E. (1977). The unheard cry for meaning (M. Tabrizi, \& A. Alavi Nia, Persian Trans.). Tehran: Fararavan Publication.

Ghanbari, S., Amani, A., Namdari Pezhman, M., Bidi, F., Kareshki, H. (2012). Structural analysis of relationship of internet addiction with depression, social adjustment and self-esteem. Scientific Journal of Hamadan University of Medical Sciences, 19(3), 41-8.

He, F., Zhou, Q., Li, J., Cao, R., \& Guan, H. (2014). Effect of social support on depression of internet addicts and the mediating role of loneliness. International Journal of Mental Health Systems, 8(1), 34. doi: 10.1186/1752-4458-8-34

Hooman, H. A. (2012). [Structural equations modeling in LISREL (Persian)]. Tehran: SAMT.

Jafari, N., \& Fatehizadeh, M. (2012). [Investigation of the relationship between internet addiction and depression, anxiety, stress and social phobia among students in Isfahan University (Persian)]. Scientific Journal of Kurdistan University of Medical Sciences, 17(4), 1-9.

Khoshakhlagh, H., \& Faramarzi, S. (2012). The relationship of emotional intelligence and mental disorders with internet addiction in internet users university students. Addiction $\mathcal{E}$ health, 4(3-4), 133-41. PMCID: PMC3905545

Kleftaras, G., \& Psarra, E. (2012). Meaning in life, psychological well-being and depressive symptomatology: A comparative study. Psychology, 3(4), 337-45. doi: 10.4236/psych.2012.34048

Ko, C. H., Yen, J. Y., Yen, C. F., Chen, C. S., \& Chen, C. C. (2012). The association between Internet addiction and psychiatric disorder: A review of the literature. European Psychiatry, 27(1), 1-8. doi: 10.1016/j.eurpsy.2010.04.011

Koukia, E., Mangoulia, P., \& Alexiou, E. (2014). Internet addiction and psychopathological symptoms in Greek university students. Journal of Addictive Behaviors Therapy \& Rehabilitation, 3(3). doi: 10.4172/2324-9005.1000125

Lee, J. Y., Shin, K. M., Cho, S.-M., \& Shin, Y. M. (2014). Psychosocial risk factors associated with internet addiction in Korea. Psychiatry Investigation, 11(4), 380. doi: 10.4306/ pi.2014.11.4.380

Mashak, R. (2006). [Comparison of irrational beliefs, social support and social anxiety of female students with employed and non-em- ployed mothers in Ahvaz high schools (Persian)]. Ahvaz: Islamic Azad University of Ahvaz.

Masoudnia, E. (2012). Internet addiction and risk of sleep disorder among adolescents. Journal of Research in Behavioural Sciences, 10(5), 350-62.

Mazhari, S. (2012). The prevalence of problematic internet use and the related factors in medical students, Kerman, Iran. Addiction \& Health, 4(3-4), 87-94. PMCID: PMC3905543

Mesrabadi, J., Jafariyan, S., \& Ostovar, N. (2013). Discriminative and construct validity of meaning in life questionnaire for Iranian students. International Journal of Behavioral Sciences, 7(1), $83-90$.

Meyers, L. S., Gamst, G., \& Guarino, A. J. (2016). Applied multivariate research: Design and interpretation. Thousand Oaks, California: Sage publications.

Mincu, C. L., \& Taşcu, A. (2015). Social support, satisfaction with physician-patient relationship, couple satisfaction, body satisfaction, optimism as predictors of life satisfaction in people having a current perceived health problem. Procedia - Social and Behavioral Sciences, 187, 772-6. doi: 10.1016/j. sbspro.2015.03.164

Moiedfar, S., Habib Poor, K., \& Ganji, A. (2005). Internet addiction, causes and consequences. Rasaneh, 63, 39-68.

Mustafa, H. R., Short, M., \& Fan, S. (2015). Social support exchanges in facebook social support group. Procedia - Social and Behavioral Sciences, 185, 346-51. doi: 10.1016/j.sbspro.2015.03.449

Onsroodi, E., Hoseinian, S., Salehi, F., \& Moemeni, F. (2014) [The interrelationship of internet addiction, adolescents' conflict with parents, multidimensional perceived social support, and happiness (Persian)]. Journal of Family and Research, 10(20), 77-94.

Owens, G. P., Steger, M. F., Whitesell, A. A., \& Herrera, C. J. (2009). Posttraumatic stress disorder, guilt, depression, and meaning in life among military veterans. Journal of Traumatic Stress, 22(6), 654-7. doi: 10.1002/jts.20460

Pirzadeh, A. (2012). The relation between general health and internet addiction in scholars of Isfahan University of Medical Sciences. Zahedan Journal of Research in Medical Sciences, 13(10), 63.

Pontes, H. M., Szabo, A., \& Griffiths, M. D. (2015). The impact of Internet-based specific activities on the perceptions of Internet addiction, quality of life, and excessive usage: A crosssectional study. Addictive Behaviors Reports, 1, 19-25. doi: 10.1016/j.abrep.2015.03.002

Schulenberg, S. E., Strack, K. M., \& Buchanan, E. M. (2011). The meaning in life questionnaire: psychometric properties with individuals with serious mental illness in an inpatient setting. Journal of Clinical Psychology, 67(12), 1210-9. doi: 10.1002/ jclp.20841

Sharifi, T., Shokr Kon, H., Ahadi, H., \& Mazaheri, M. M. (2009). [Investigating the Relationship between Religious and $\mathrm{Na}$ tional Identities and Students' Mental Health (Persian)]. New Findings in Psychology, 4(11), 125-142.

Steger, M. F., Fitch Martin, A. R., Donnelly, J., \& Rickard, K. M (2014). Meaning in life and health: Proactive health orientation links meaning in life to health variables among American 
undergraduates. Journal of Happiness Studies, 16(3), 583-97. doi: 10.1007/s10902-014-9523-6

Steger, M. F., Frazier, P., Oishi, S., \& Kaler, M. (2006). The meaning in life questionnaire: Assessing the presence of and search for meaning in life. Journal of Counseling Psychology, 53(1), 80-93. doi: 10.1037/0022-0167.53.1.80

Sung, J., Lee, J., Noh, H. M., Park, Y. S., \& Ahn, E. J. (2013). Associations between the risk of internet addiction and problem behaviors among Korean adolescents. Korean Journal of Family Medicine, 34(2), 115. doi: 10.4082/kjfm.2013.34.2.115

Tang, J. H., Chen, M. C., Yang, C. Y., Chung, T. Y., \& Lee, Y. A. (2016). Personality traits, interpersonal relationships, online social support, and Facebook addiction. Telematics and Informatics, 33(1), 102-8. doi: 10.1016/j.tele.2015.06.003

Woo, C. R. S., \& Brown, E. J. (2013). Role of meaning in the prediction of depressive symptoms among trauma-exposed and nontrauma-exposed emerging adults. Journal of Clinical Psychology, 69(12), 1269-83. doi: 10.1002/jclp.22002

$\mathrm{Wu}, \mathrm{C} . \mathrm{H}$. (2004). The role of social support on internet addiction. Journal of Cyber Culture and Information Society, 7, 173-89.

Xu, J., Shen, L., Yan, C., Hu, H., Yang, F., Wang, L., et al. (2012). Personal characteristics related to the risk of adolescent internet addiction: A survey in Shanghai, China. BMC Public Health, 12(1):1106. doi: 10.1186/1471-2458-12-1106 
